\title{
Stimulation of acupoint P6 before induction of anesthesia to prevent postoperative nausea and vomiting; a randomized control trial
}

\author{
Saman Hamid ${ }^{1}$, Mohsin Nazir Butt ${ }^{2}$, Azhar Rehman ${ }^{2}$, Gauhar Afshan ${ }^{2}$ \\ Author affiliation: \\ 1. Medical College, Aga Khan University Faculty of Health Sciences, Stadium Road, Aga Khan University Hospital, Karachi, \\ Karachi City, Pakistan 74800. \\ 2. Department of Anesthesiology, The Aga Khan University Hospital, Stadium Road, Aga Khan University Hospital, Karachi, \\ Karachi City, Pakistan 74800. \\ Correspondence: Dr. Saman Hamid (ORCID-0000-0002-7023-2136); E-mail: saman.hamid@scholar.aku.edu; Phone: \\ $+923430475661$
}

\section{Abstract}

Background \& Aims: Laparoscopic surgery has been associated with troublesome postoperative nausea and vomiting (PONV) in patients. Various regimens have been tried by the anesthesiologist to prevent PONV in laparoscopic cholecystectomy (LapChole). The primary objective of this study was to determine the role of stimulation of acupoint P6 for this purpose in patients undergoing LapChole at our institution.

Methodology: A randomized clinical trial was conducted at the Aga Khan University Hospital, Karachi, Pakistan. The research setting took place at the Post Anesthesia Care Unit (PACU) of the hospital. A total of 84 participants undergoing LapChole, ASA Status I or II, were enrolled and randomly allocated into two groups: 41 in control and 43

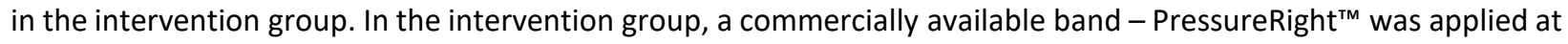
the wrist and the beads were placed exactly at P6 point. In the control group, the band was applied at the wrist with the beads placed on the dorsal surface. In both groups, acupressure band was applied before the induction of anesthesia in the waiting area of the operating room and continued during the intraoperative period and six hours postoperatively.

Measurements: Postoperatively, patients were monitored for postoperative nausea and vomiting at the time of arrival in post anesthesia care unit [PACU], after half hour, three hour and six hours postoperatively using numerical pain scale [NPS] from 1-10 [1=none, 2-5=mild, 6-7=moderate, 8-10=severe] for PONV separately.

Main Results: The results of PONV in PACU, at 30 minutes, three hours and six hours postoperatively showed an insignificant difference in intervention and control group. The frequencies of mild to moderate PONV were gradually reduced in both groups but the reduction was more pronounced in the control group than in the intervention group. There were no reported events of severe PONV at three hours and six hours postoperatively in both groups. The use of rescue antiemetics was statistically insignificant between the two groups $[P=0.744]$.

Conclusions: Our study reports that acupressure at Neiguan P6 point starting before the induction of anesthesia till 6 hours postoperative has no significant role in preventing PONV in patients undergoing laparoscopic cholecystectomy. Rescue antiemetics were required in both groups with a similar frequency.

Key words: Acupoint P6; Acupressure; PONV; Laparoscopic cholecystectomy; Antiemetics; Randomized Clinical Trial Ethical approval: AKU No. 5365-ane-ERC-18, dated July 17, 2018

Citation: Hamid S, Butt MN, Rehman A, Afshan G. Stimulation of acupoint P6 before induction of anesthesia to prevent postoperative nausea and vomiting; a randomized control trial. Anaesth. pain intensive care 2022;26(1):96101. DOI: $10.35975 / a p i c . v 26 i 1.1774$

Received: August 6, 2021, Reviewed: October 22, 2012, Accepted: December 15, 2021 


\section{Introduction}

All surgical interventions requiring anesthesia carry a risk of postoperative complications. One of the most common complications, second to pain, is postoperative nausea and vomiting (PONV). ${ }^{1,2}$ The general incidence of nausea and vomiting has been reported to be $50 \%$ following anesthesia and $30 \%$ following surgery respectively. While this percentage in high-risk patients, can go as high as $80 \% .^{3}$ Postoperative nausea and vomiting is a stressful and uncomfortable condition for patients. Unresolved PONV may lead to long stay in post anesthesia care unit (PACU) and unanticipated hospital admission, resulting in a significant increase in overall health care costs and negative effect on patient satisfaction. ${ }^{4}$ Considering these facts, it is essential for every anesthesiologist to aim for a PONV free patient in postoperative period. 5

It has been shown that aggressive prophylaxis measures, combined with changes in anesthetic technique, can reduce the incidence of PONV. Currently, pharmacological prophylaxis is widely used in clinical practice however at present no therapy is absolutely effective in preventing PONV. Additionally, medical therapy can be expensive and be associated with many adverse effects. ${ }^{5}$ Since it has been established that current antiemetics and anesthetic techniques are not sufficient in reducing PONV, the use of supplemental methods for prophylaxis has become widespread and accepted. These supplemental strategies include hypnosis, acupuncture, acupressure, relaxation techniques, behavior therapy, and guided imagery. ${ }^{6}$

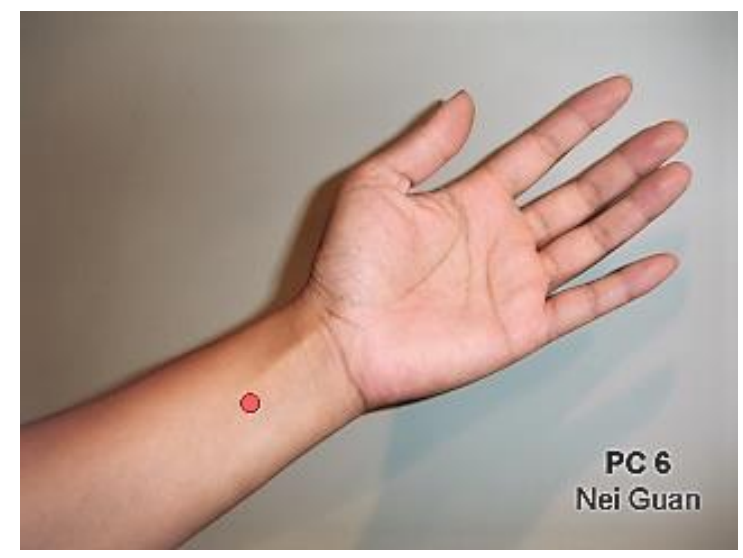

Figure 1: Pericardium point P6

Neiguan acupoint (P6) (Figure 1) is commonly used to help relieve nausea, vomiting, motion sickness, carpal tunnel syndrome, and headaches. ${ }^{7}$ It is located three finger breadths below the wrist on the inner forearm in between the two tendons and has been cited in many research studies that the stimulation acupoint P6 helps in preventing PONV. Neiguan acupoint can be stimulated

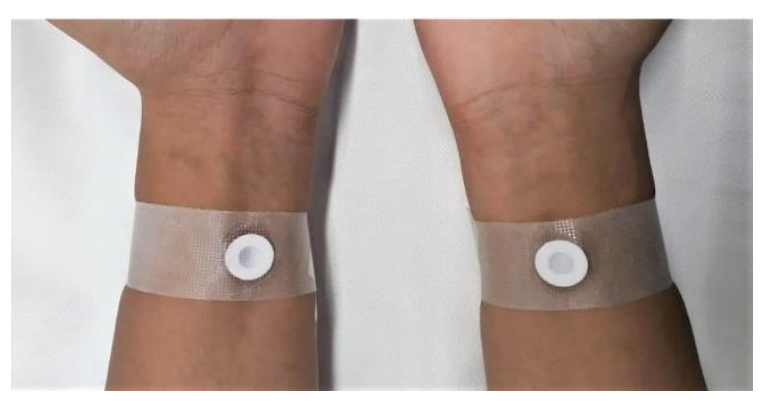

Figure 2: Acupressure band placement at P6 in intervention group

by various techniques. Acupressure is non-invasive method induced by manual stimulation and is devoid of complications like nerve damage and infection transmission. ${ }^{7,8}$ Acupressure wrist bands are less expensive compared to most antiemetics and the adverse effects of acupoint stimulation are minimal. ${ }^{9}$ Studies have reported the efficacy of using Acupressure in significantly improving postoperative nausea and vomiting, ${ }^{10-12}$ while there have also been reports of no significant difference. ${ }^{13,14}$

Laparoscopic cholecystectomy is one of the most common procedures performed worldwide in general surgery. ${ }^{15}$ While it is a fairly simple procedure with minimal risk of infection as compared to laparotomy, there is a high risk of postoperative nausea and vomiting due to the surgical technique involved, type of anesthesia used, and prior risk factors of the patients.

The primary objective of this study was aimed to determine the role of stimulation of Acupoint P6 for preventing PONV in patients undergoing laparoscopic cholecystectomy. The requirements of rescue antiemetics and frequency of their adverse effects were also looked at.

\section{Methodology}

\section{Study Design:}

This was a randomized controlled trial (RCT). After approval from the ethics review committee and clinical trials unit it was conducted in the operating rooms, postoperative anesthesia care unit and surgical wards of Aga Khan University Hospital. A total of 84 patients undergoing laparoscopic cholecystectomy were enrolled. The sample size was based on having $80 \%$ power ( $\alpha$ error of $5 \%$ ) to detect a $50 \%$ reduction in risk of PONV in patients who will receive $\mathrm{P} 6$ acupoint stimulation (given an estimated $30 \%$ to $60 \%$ rate of PONV in the control group). Sampling was done through non-probability purposive sampling.

The inclusion criteria were adult patients aged 18-50 y, of either gender, and belonging to American Society of Anesthesiology (ASA) status I or II for undergoing 


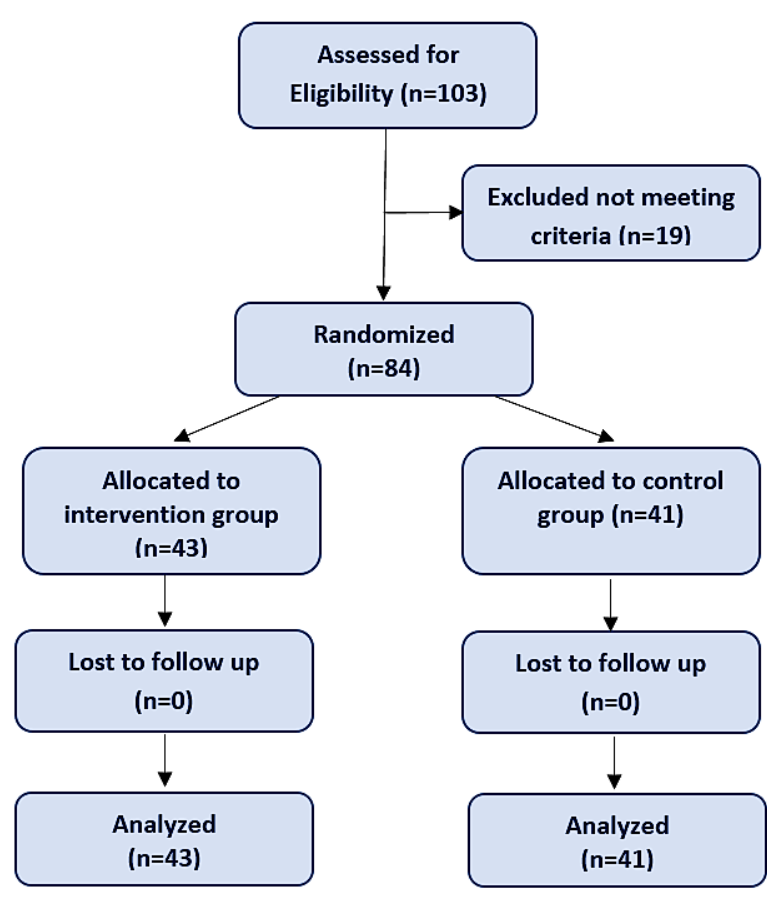

Figure 3: CONSORT flow diagram for the study

laparoscopic cholecystectomy. The exclusion criteria were patients who refused the consent and with a history of wrist surgery, gastro-esophageal surgery, esophageal reflux and any skin disease. Pregnant Patients and patients with more than one antiemetic used intraoperatively were also excluded.

Study participants were randomized into two groups, i.e. correct acupressure site (intervention) and incorrect acupressure site (control) by block randomization. Sequence generation and allocation concealment were computer generated and done by clinical trial unit. Acupressure was applied by a pre-formed purposively made wrist band in both hands. In this study we used a wrist band with the name of 'Pressure Right@', manufactured for Pressure Point Inc. (36 Green Meadow Drive, USA). Each band had a bead at one end and sticking surface on the other end. In the intervention group, the band was applied at the wrist and the beads were placed exactly at P6 point (Figure 2). In the control group, the band was applied at the wrist with the beads placed on the dorsal surface. In both groups, acupressure band was applied before the induction of anesthesia in the waiting area of the operating room and continued during intraoperative period and six hours postoperatively.

In operating room, general anesthesia was given with Nalbuphine $0.1 \mathrm{mg} / \mathrm{kg}$, propofol $2 \mathrm{mg} / \mathrm{kg}$ and atracurium $0.5 \mathrm{mg} / \mathrm{kg}$. Endotracheal intubation using murphy eyed cuffed PVC tube was done through conventional or video laryngoscopy. Anesthesia was maintained using isoflurane and intermittent use of atracurium. Injection metoclopramide (Maxolon®) $0.1 \mathrm{mg} / \mathrm{kg}$ was given 15 min prior to the extubation (as per discretion of the primary anesthetist in the operating room) Neostigmine $2.5 \mathrm{mg}$ and glycopyrrolate $0.2 \mathrm{mg}$ were administered as reversal agent after getting train of four. Trachea was extubated when the patients fulfilled the extubation criteria. Postoperatively, participants were shifted to the recovery room with wrist band for monitoring. The bands were removed in the ward at six hours postoperatively.

Postoperatively, patients were monitored for postoperative nausea and vomiting at the time of arrival in post-anesthesia care unit (PACU), at half hour, three hours and six hours postoperatively, using a $10 \mathrm{~cm}$ numerical pain scale (NPS) marked from 1 to $10(1=$ none, $2-5=$ mild, $6-7=$ moderate, $8-10=$ severe $)$ for nausea as well as vomiting separately. In addition, routine postoperative pain scores were also monitored.

\section{Results}

A total of 84 participants were enrolled and randomly allocated into 2 groups: 41 in control and 43 in intervention group. None of the participants was excluded and data was analyzed by the intention to treat analysis principle. (Figure 3). No significant statistical difference was found between the control and intervention group in terms of age, sex, ASA physical status and co-morbid conditions (Table1).

Doses of anesthesia induction agents, muscle relaxants, analgesics and antiemetic used during surgery were also comparable (Table-2). The mean pain score in post anesthesia care unit (PACU), 30 minutes, three hours and six hours postoperatively were also comparable between the intervention and control groups (Table 3).

The main outcome of trial was PONV, measured by visual analogue scale. . The results of PONV in PICU, at 30 minutes, three hours and six hours postoperatively showed an insignificant difference in intervention and control group. In PICU, about $72 \%$ of patients in intervention group and $85 \%$ of patients in control group developed mild to moderate PONV. The frequencies of mild to moderate PONV were gradually reduced in both groups but the reduction was more pronounced in control group than in the intervention group. At six hours postoperatively, the frequency of mild to moderate PONV in intervention group was about $42 \%$ while in control group it came out to be around $26.8 \%$. The frequency of severe PONV was similar at first two time points (i.e. at PACU and 30 minutes postoperatively) in both groups (i.e. $7 \%$ in intervention group \& $4.9 \%$ in control group at PACU and 30 minutes postoperatively). There were no reported events of severe PONV at three hours and six hours postoperatively in both groups (Table 4). About $2.3 \%$ of patients in intervention group 


\begin{tabular}{|c|c|c|c|c|}
\hline \multicolumn{2}{|l|}{ Variables } & $\begin{array}{l}\text { Intervention } \\
\text { Bead } \\
(n=43)\end{array}$ & $\begin{array}{l}\text { Control } \\
\text { Bead } \\
(n=41)\end{array}$ & P-Value \\
\hline \multicolumn{2}{|c|}{ Age (Years) } & $38.05 \pm 8.12$ & $\begin{array}{l}37.56 \pm \\
8.34\end{array}$ & 0.787 \\
\hline Gender & $\begin{array}{l}\text { Female } \\
\text { Male }\end{array}$ & $\begin{array}{l}28(65.1) \\
15(34.9)\end{array}$ & $\begin{array}{l}34(82.9) \\
7(17.1)\end{array}$ & 0.063 \\
\hline $\begin{array}{l}\text { ASA- } \\
\text { Status }\end{array}$ & $\begin{array}{l}\text { I } \\
\text { II } \\
\text { III }\end{array}$ & $\begin{array}{l}19(44.2) \\
24(55.8) \\
0(0)\end{array}$ & $\begin{array}{l}21(51.2) \\
19(46.3) \\
1(2.4)\end{array}$ & 0.442 \\
\hline $\begin{array}{l}\text { Co- } \\
\text { morbids }\end{array}$ & $\begin{array}{l}\text { Hypertension } \\
\text { Diabetes Mellitus } \\
\text { IHD } \\
\text { COPD } \\
\text { Others }\end{array}$ & $\begin{array}{l}10(23.3) \\
3(7) \\
0(0) \\
0(0) \\
8(18.6)\end{array}$ & $\begin{array}{l}1(2.4) \\
8(19.5) \\
1(2.4) \\
4(2.4) \\
11(26.8)\end{array}$ & $\begin{array}{l}0.005 \\
0.089 \\
0.488 \\
0.488 \\
0.368\end{array}$ \\
\hline Data prese & d as $n(\%)$ & & & \\
\hline
\end{tabular}

\begin{tabular}{|c|c|c|c|}
\hline Variables & $\begin{array}{l}\text { Intervention Bead } \\
(n=43)\end{array}$ & $\begin{array}{l}\text { Control Bead } \\
(n=41)\end{array}$ & P-value \\
\hline $\begin{array}{l}\text { Combination of } \\
\text { nalbuphine, propofol and } \\
\text { atracurium used }\end{array}$ & $42(97.7)$ & $41(100)$ & 0.326 \\
\hline $\begin{array}{l}\text { Inj. Maxolon } 0.1 \mathrm{mg} / \mathrm{kg} 15 \\
\text { min before extubation }\end{array}$ & $28(66.7)$ & $29(70.7)$ & 0.690 \\
\hline Rescue analgesia given & $43(100)$ & $41(100)$ & NA \\
\hline Ketorolac & $24(55.8)$ & $19(46.3)$ & 0.385 \\
\hline Paracetamol & $29(67.4)$ & $22(53.7)$ & 0.196 \\
\hline Tramadol & 7 (16.3) & $12(29.3)$ & 0.155 \\
\hline Data presented as $n(\%)$ & & & \\
\hline
\end{tabular}

Table 3: Comparison of mean pain score between groups with respect to time

\begin{tabular}{llll} 
Time & Intervention bead & Control bead & P-value \\
In PICU & $3.30 \pm 2.445$ & $3.22 \pm 2.031$ & 0.867 \\
$30 \mathrm{~min}$ & $3.14 \pm 2.484$ & $3.10 \pm 2.131$ & 0.934 \\
3 hours & $1.09 \pm 1.493$ & $.83 \pm 1.548$ & 0.429 \\
6 hours & $.95 \pm 1.327$ & $.61 \pm 1.159$ & 0.211 \\
\hline \multicolumn{2}{l}{ Data presented as Mean $\pm S D$} & & \\
\hline
\end{tabular}

5a), the odds ratio of PONV was statistically insignificant $(\mathrm{OR}=0.408$ $95 \% \mathrm{CI}=0.115$ to 1.449$). . \mathrm{In}$ multivariable logistic regression, the association of intervention bead came out to be marginally insignificant $\left(\mathrm{Chi}^{2}\right.$ $=12.55, \mathrm{P}=0.05$ ), after adjusting for age, sex, intraoperative and postoperative antiemetic used (Table $5 b)$.

\section{Discussion}

In current surgical practices, high incidences of PONV up to 53-72\% have been reported with laparoscopic cholecystectomy. ${ }^{15}$ Although it was widely investigated in the last two decades, but we are still unable to find effective treatment for prevention of PONV. The modern medicine is now looking for alternate ways of reducing this postoperative complication. Acupressure has recently been introduced in anesthetics practice. It has no side effects or drug interactions, is non-invasive, simple to apply, has a high degree of patient acceptance and is economical. ${ }^{7}$

While some research studies have shown beneficial effects of acupressure stimulation in preventing PONV but it is still inconsistent in giving desired results. ${ }^{7}, 10,12$ This current study was done to determine the role of prophylactic use of Acupoint P6 stimulation for prevention of PONV and the need for rescue antiemetics in patients undergoing laparoscopic cholecystectomy.

Our study reports that acupressure at Neiguan P6 point starting before the induction of anesthesia till 6 hours postoperative has no significant role in preventing PONV in patients and $4.9 \%$ of patients in control group, developed two episodes of vomiting in first six hours postoperatively. Rescue antiemetics were used in $67.4 \%$ of intervention group and $70.7 \%$ of control groups; the use of rescue antiemetics was statistically insignificant between the two groups $(\mathrm{P}=0.744)$.

Logistic regression was also used to predict the occurrence of PONV in two groups and to develop prediction model with adjusting different plausible covariates (Table 5). In binary logistic regression (Table undergoing laparoscopic cholecystectomy. The authors have done the similar study previously with the manual bead placement (compared to recent wrist bands) with the similar results too. ${ }^{14}$ In our previous study, we used acupressure on one hand only and that was the major criticism and it was suggested to use acupressure in both hands that we did in this study.

It is important to note that in our current study, medical intervention needed to be done in both study groups. The rescue anti-emetics were given in 60 patients (out of 84 


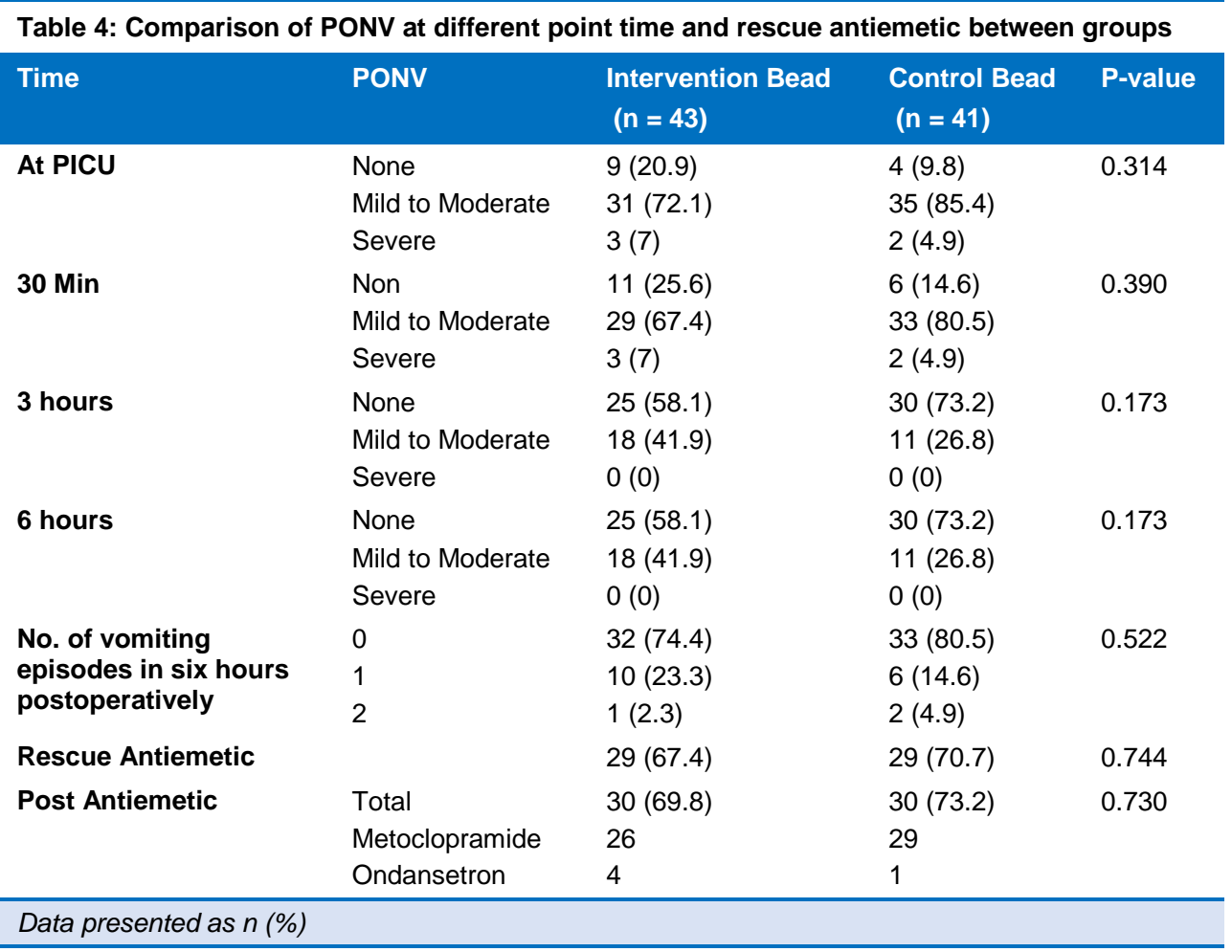

The strength of this study is that it is a randomized control trials with stringent inclusion and exclusion criteria and we choose only single surgical intervention to avoid biases. Our study does have certain limitations. As it is a single-centered study with a relatively small sample size. Since the wrist band placement and data collection were done by different research assistant (although trained), there is a possibility of difference in skill for the band placement and PONV reporting biases. This highlights the importance of patients) with no significant difference between the control and intervention groups. The rescue antiemetic drugs used postoperatively included Metoclopramide (91\% of patients) and Ondansetron (9\% of patients).

A wide variety of factors influence the incidence of PONV in laparoscopic cholecystectomy and biases from these must be eliminated from a study for the management of postoperative nausea and/or vomiting to draw valid conclusions. Risk factors such as age and sex of patient, obesity, history of postoperative nausea or vomiting or motion sickness, duration of surgery, the use of anesthetic drugs, especially inhalational agents and the opioids may contribute to these episodes. We controlled these risk factors within the study design. Duration of anesthesia and surgery and anesthetic drugs used were similar in both groups. The demographics like age, weight and sex distributions were also similar in both groups. In addition, we did logistic regression for adjusting different plausible factors in case was have missed any in study design.

One possible explanation of our study result is that PONV (being a subjective experience often) is over reported in our population as they often are not properly counselled about pain \& PONV in pre-operative period. In addition, their own believe, that only medical interventions relieve the PONV, can be the reason of over -reporting of PONV requiring anti-emetics.

overcoming limitations of the study in advance to avoid such biases that may influence the overall outcome.

\section{Conclusion}

Our study reports that acupressure at Neiguan P6 point starting before the induction of anesthesia till 6 hours postoperative has no significant role in preventing PONV in patients undergoing laparoscopic cholecystectomy. Rescue antiemetics were required in both groups with the similar frequency.

\section{Conflict of interest}

None declared by the authors.

\section{Acknowledgement}

The authors are thankful to Mr. Joseph DiLustro, the inventor of Pressure Right ${ }^{\circ}$ bands and owner of Pressure Point Inc. USA to kindly provide the devices free of cost for the study purpose.

We are also thankful to the Editor-in-Chief of 'Anaesthesia, Pain \& Intensive Care' journal to facilitate this research.

\section{Authors' contribution}

All authors participated in the concept, conduct of the study, data collection, statistical analysis and manuscript preparation. 


\section{References}

1. Chung JW, Lui JC. Postoperative pain management: study of patients' level of pain and satisfaction with health care providers' responsiveness to their reports of pain. Nurs Health Sci. 2003;5(1):13-21. [PubMed] DOI: 10.1046/j.14422018.2003.00130.x

2. Moysés AM, Durant LC, Almeida AM, Gozzo TO. Integrative review of factors related to the nursing diagnosis nausea during antineoplastic chemotherapy. Rev Lat Am Enfermagem. 2016;24:e2812. [PubMed] DOI: 10.1590/1518-8345.1176.2812

3. Gan TJ, Diemunsch P, Habib AS, Kovac A, Kranke P, Meyer $\mathrm{TA}$, et al. Consensus guidelines for the management of postoperative nausea and vomiting. Anesth Analg. 2014;118(1):85-113. [PubMed] DOl: 10.1213/ANE.0000000000000002

4. Kranke P, Eberhart LH. Possibilities and limitations in the pharmacological management of postoperative nausea and vomiting. Eur J Anaesthesiol. 2011 Nov;28(11):758-65. [PubMed] DOI: 10.1097/EJA.0b013e32834a4e1e

5. Le TP, Gan TJ. Update on the management of postoperative nausea and vomiting and postdischarge nausea and vomiting in ambulatory surgery. Anesthesiol Clin. 2010 Jun;28(2):22549. [PubMed] DOI: 10.1016/j.anclin.2010.02.003

6. Canakci E., Catak T. Postoperative nausea and vomiting: risks, prophylaxis, non-drug alternative methods. Middle Black Sea J Health Sci. 2019; 5(3): 284-292. DOI: 10.19127/mbsjohs.637394

7. Unulu M, Kaya N. The effect of neiguan point (p6) acupressure with wristband on postoperative nausea, vomiting, and comfort level: a randomized controlled study. J Perianesth Nurs. 2018;33(6):915-27. [PubMed]

DOI:
8. Lee A, Fan LT. Stimulation of the wrist acupuncture point P6 for preventing postoperative nausea and vomiting. Cochrane Database Syst Rev. 2009 Apr 15;(2):CD003281. [PubMed] DOI: 10.1002/14651858.CD003281.pub3

9. Zhang Y, Zhang C, Yan M, Wang N, Liu J, Wu A. The effectiveness of PC6 acupuncture in the prevesntion of postoperative nausea and vomiting in children: A systematic review and meta-analysis. Paediatr Anaesth. 2020;30(5):55263. DOI: $10.1111 /$ pan. 13860

10. Bapat V, Gaikwad S. Acupressure for prevention of postoperative nausea and vomiting. Indian J Appl Res. 2016:6(3). [FreeFullText]

11. Eslami J, Darvishi I, Ebrahimi A, Akbarzadeh M. Effect of acupressure in Pericardium 6 acupoint on nausea and vomiting after general surgery. Nurs Midwifery Stud. 2019;8:143-8. DOI: 10.4103/nms.nms_4_18

12. Fan CF, Tanhui E, Joshi S, Trivedi S, Hong Y, Shevde K. Acupressure treatment for prevention of postoperative nausea and vomiting. Anesth Analg. 1997 Apr;84(4):821-5. [PubMed] DOI: 10.1097/00000539-199704000-00023

13. Yilmaz Sahin S, lyigun E, Can MF. Effect of acupressure application to the P6 acupoint before laparoscopic cholecystectomy on postoperative nausea-vomiting: A randomized controlled clinical study. Int J Nurs Stud. 2018 Nov;87:40-48. [PubMed] DOI: 10.1016/j.jinurstu.2018.07.011

14. Samad K, Afshan G, Kamal R. Effect of acupressure on postoperative nausea and vomiting in laparoscopic cholecystectomy. J Pak Med Assoc. 2003 Feb;53(2):68-72. [PubMed]

15. Brenner P, Kautz DD. Postoperative Care of Patients Undergoing Same-Day Laparoscopic Cholecystectomy. AORN J. 2015 Jul;102(1):16-29. [PubMed] DOI: 10.1016/j.aorn.2015.04.021 